\title{
CONCORDANCIA DE LA TOMA DE PRESIÓN ARTERIAL ENTRE EL PERSONAL DE ENFERMERÍA Y EL MÉDICO
}

José Ignacio Hernández, MD. ", Omar Oliveros, MD. ", Eliana Vanegas, MD.

\section{Resumen}

Introducción: la tensión arterial (TA) es uno de los parámetros usados para determinar el estado hemodinámico de un paciente. Cuando la encontramos alterada nos vemos obligados a tomar conductas que pueden ser erróneas y económicamente significativas si la medición fue incorrecta. Este trabajo desea resaltar la importancia que tiene la toma de la TA con una técnica precisa y aclarar si las diferencias en las mediciones realizadas por el médico y la enfermera corresponden a inconvenientes técnicos por los tensiómetros, $o$ si por el contrario nuestro personal no aplica las técnicas adecuadas.

Métodos: ingresaron al estudio 592 pacientes mayores de 18 años, hospitalizados en los diferentes pabellones del Hospital de San José, sin importar su condición clínica ni su género. Se anotó la TA determinada por el personal de enfermería y luego, con el mismo tensiómetro, por parte de los médicos internos. Después el residente de Medicina Interna lo hizo con un tensiómetro estándar calibrado. Para el análisis estadístico se usó el método de concordancia, a través del coeficiente de correlación de Pearson. Además se obtuvo el estimativo puntual con su respectivo intervalo de confianza.

Resultados: a 592 paciente se les tomó cada 10 minutos la TA en tres ocasiones y se demostró que las cifras variaron según quien ejecutó la medición. La diferencia fue más notoria al comparar las cifras del residente y la enfermera, obteniendo una diferencia de más de $10 \mathrm{~mm}$ Hg en la TA sistólica en 29\% de los casos (IC 0,260 - 0,334) y en la diastólica en el 30\% (IC 0,267 - 0,341), con un coeficiente de concordancia entre 0,78 y 0,91 .

Conclusiones: teniendo en cuenta que el residente utilizó la técnica en forma precisa y dadas las diferencias tan marcadas en las cifras obtenidas, se deduce que la forma en que se midió la TA no es adecuada. Por tanto, es imperativo revisar el procedimiento e instruir al personal médico y paramédico sobre la manera correcta de tomarla.

\section{Introducción}

La hipertensión arterial es una de las enfermedades más frecuentes en el mundo entero y también una de las que mayores complicaciones genera. Cualquier medida que se adopte desde el punto de vista terapéutico pensando que el paciente la sufre, rotula al individuo como hipertenso, lo cual implica un cambio radical en sus hábitos de vida.

El registro de la TA es uno de los parámetros usados para aproximarse al estado hemodinámico de un sujeto. Cuando se encuentra elevada es necesario tomar una serie de conductas, las cuales pueden estar equivocadas y tener un impacto negativo en la esfera econó-

Departamento de Medicina Interna, Hospital de San José - Sociedad de Cirugía de Bogotá. mica si la medición no fue realizada en forma adecuada. De ahí la importancia de una óptima determinación por parte del personal médico y paramédico, para evitar decisiones terapéuticas erradas, que pueden ser deletéreas para el paciente y aumentan los costos en nuestro sistema de salud.

Se desea resaltar la importancia de una toma de TA con una técnica precisa y aclarar si las diferencias en las mediciones realizadas por el médico y la enfermera corresponden a inconvenientes técnicos por problemas con los tensiómetros, o si por el contrario nuestro personal de enfermería no aplica las técnicas adecuadas.

\section{Tensión arterial}

La presión de la sangre dentro del sistema arterial puede medirse en forma directa al introducir un catéter 
dentro de una arteria. En la práctica clínica rutinaria no es posible utilizar este método directo y el médico se encuentra obligado a estimarla mediante uno indirecto, que consiste en contraponer a la fuerza de la sangre una masa de aire a presión, que luego es determinada mediante un manómetro de mercurio o uno aneroide.

El aparato para medir la tensión arterial es el tensiómetro. Está compuesto por un manguito de caucho de por lo menos $12 \mathrm{~cm}$ de ancho, cubierto en su totalidad por una tela fuerte inextensible. Dicho manguito se encuentra conectado por un extremo con el manómetro a través de un tubo, y por el otro se halla en conexión con una pera de caucho para inflarlo. Una pequeña válvula colocada entre la pera y el manguito permite el escape de aire y reducir a voluntad la presión dentro del manguito. Los manómetros de mercurio no se descalibran, pero los aneroides tienen un sistema que se descompone con el uso y por eso necesitan ser calibrados en forma periódica, comparándolos con uno de mercurio.

\section{Técnica para tomar la tensión}

El manguito desinflado se ajusta alrededor del brazo, procurando que su borde inferior quede unos dos $\mathrm{cm}$ por encima del pliegue del codo. Una vez colocado en esta posición es inflado hasta que la presión del aire que contiene supere a la sanguínea y oblitere la arteria humeral, lo cual es fácil de reconocer porque desaparece el pulso radial. A continuación se aumenta la presión un poco más para luego, accionando la válvula, permitir lentamente la salida del aire y reducir la presión dentro del manguito de manera gradual, hasta que la presión sanguínea supera la presión del aire y la sangre comienza a fluir en pequeñas cantidades hacia los tejidos distales. Lo anterior corresponde a las débiles pulsaciones percibidas en la arteria radial. En ese momento la presión que indica el manómetro representa la presión sistólica. Existen dos métodos para tomar la presión arterial: el auscultatorio y el palpatorio.

Método auscultatorio: Este método se basa en el hecho de que al auscultar la arteria humeral más allá del borde inferior del manguito, se oyen ciertos sonidos a medida que se desinfla. Estos son utilizados como indicadores de la tensión sistólica y diastólica.
Método palpatorio: Se insufla el manguito aumentando la presión un poco por encima de la cifra que coincide con la desaparición del pulso radial. A continuación el examinador comienza a desinflarlo lentamente y obtiene la presión sistólica cuando comienza a detectar de nuevo el pulso, asumiendo que los primeros escapes de sangre por debajo del manguito, los cuales se perciben muy bien en la auscultación, también ocasionan una pulsación perceptible. Lo más probable, sin embargo, es que ese pequeño flujo no alcance a producir una onda pulsátil. Por eso el método palpatorio proporciona lecturas sistólicas más bajas (aproximadamente unos $10 \mathrm{~mm} \mathrm{Hg}$ ), con respecto a las obtenidas con el método auscultatorio. Una desventaja seria del método palpatorio es que no permite apreciar la presión diastólica.

\section{Normas generales para tomar la TA}

La persona debe estar sentada o en decúbito dorsal, con el brazo ligeramente flexionado a la altura del corazón, y apoyado con firmeza sobre una superficie plana. El brazo y el antebrazo tienen que estar desnudos o, al menos, el examinador debe cerciorarse de que las prendas de vestir no ejerzan compresión inadecuada. El manguito desinflado debe tener un ancho de 12 a $13 \mathrm{~cm}$ y se debe enrollar de manera uniforme en el brazo, lo más ajustado posible. El borde inferior se coloca por lo menos dos $\mathrm{cm}$ por encima del pliegue del codo. El estetoscopio es ubicado sin hacer demasiada presión sobre el sitio donde el examinador palpa la arteria humeral, evitando en lo posible que haga contacto con el manguito para suprimir ruidos adventicios. Una causa de error puede ser la desigualdad relativa entre el tamaño del brazalete y el diámetro de la extremidad. En brazos muy obesos por lo regular se obtienen valores elevados falsos y en brazos muy delgados cifras equivocadamente por debajo de lo normal. La aplicación demasiado floja del manguito genera datos por encima de lo normal.

Es importante que el individuo permanezca en reposo durante tiempo suficiente (entre 10 y 15 minutos) para eliminar la influencia de algún ejercicio previo o cualquier estado emocional. Es aconsejable tomarla en ambos brazos (en especial si al examinador le ha parecido 
que los pulsos radiales son asimétricos), pues en casos de aneurisma aórtico o de otros síndromes del arco aórtico, puede existir una diferencia marcada entre los dos brazos. No olvidar sin embargo que en el individuo normal puede haber una diferencia inferior a $10 \mathrm{~mm} \mathrm{Hg}$ entre uno y otro brazo.

No es necesario tomar la tensión de manera rutinaria en los miembros inferiores. Como norma, la tensión es más alta en las extremidades inferiores que en las superiores. Si se sospecha alguna entidad que invierta esta relación, por ejemplo la coartación de la aorta, lo procedente es hacer una cuidadosa palpación de los pulsos femorales. Si están disminuidos o ausentes, ello es suficiente para plantear la posibilidad de coartación. Ante la duda conviene tomar la TA en los miembros inferiores, pero recordando que si estos exceden cierto diámetro, es necesario utilizar un manguito más ancho con respecto al utilizado para los miembros superiores.

\section{Análisis estadístico}

Se utilizó el método de concordancia. Lin describió el coeficiente de concordancia y correlación, el cual determina qué tanto se desvían los datos obtenidos con los dos métodos, de la línea de perfecta concordancia de 45 grados que comienza en 0 . Tal coeficiente combina dos dimensiones: precisión y exactitud, y tiene un rango de -1 a +1 . Cuanto más está alterada la concordancia, menor será el valor del coeficiente; y sería igual a 1,0 si hay perfecta concordancia. Si esto sucede, es decir, cuando hay exactitud, los datos están cerca de la línea de perfecta concordancia. Para evaluar la precisión, se determina cuán esparcidos están los datos alrededor de dicha línea.

El coeficiente de concordancia puede dividirse en dos componentes: el producto del coeficiente de correlación, que mide precisión, y un factor de corrección de sesgo que mide exactitud, es decir, qué tanto desplazamiento de los resultados existe con respecto a la línea de 45 grados. Este coeficiente debe ser usado en conjunto con los límites de concordancia, pues son pruebas complementarias.

Los límites de concordancia indican cuán cercano es el acuerdo de las mediciones de los dos métodos, pero no proporciona una evaluación sobre qué tanto se desvían los datos de una concordancia perfecta. El coeficiente de concordancia otorga esta información, además de los datos de precisión. Debe demostrarse que dos o más métodos diferentes para hacer la misma medición, proporcionan resultados idénticos. Es indispensable el uso de pruebas estadísticas que permitan determinar cuánta coincidencia hay entre valores, es decir, si existe o no concordancia. El uso de los límites de concordancia, de fácil cálculo, permite decidir basándose en datos clínicos, si los métodos pueden ser intercambiables. El uso del conjunto con los límites de concordancia, permite determinar de una manera comprensiva si las técnicas evaluadas producen resultados similares.

\section{Coeficiente de correlación (r de Pearson)}

Cuando la variable utilizada es continua y existen dos observaciones (instrumentos u observadores) por sujeto, resulta intuitivo representar cada par de observaciones en un diagrama de puntos, examinar gráficamente si existe relación lineal entre las dos variables y calcular el coeficiente de correlación $(r)$. Se espera que cuanto más coincidan las mediciones, más se aproximará a una recta en el diagrama de puntos.

\section{Método de Bland - Altman}

Este método tiene una gran difusión para analizar la concordancia entre dos procedimientos que utilizan las mismas unidades de medida. Consiste en representar gráficamente la diferencia entre las dos observaciones contra su medida. Ello permite examinar en forma rápida la magnitud de las discrepancias y su relación con la medición. Además se puede estimar el error estándar de las diferencias y las bandas de confianza, entre las que cabe esperar que se encuentre el $95 \%$ de las diferencias.

\section{Materiales y métodos}

Ingresaron al estudio 592 pacientes mayores de 18 años, que se encontraban hospitalizados en los diferentes pabellones del Hospital de San José, sin importar su condición clínica ni su género. Se anotó la TA tomada 
por el personal de enfermería con tensiómetro de mercurio, y luego, con el mismo instrumento, fue registrada por los médicos internos. Después el residente de Medicina Interna la tomó con un tensiómetro aneroide estándar, perfectamente calibrado, sin conocer los resultados de las mediciones realizadas por el interno y la enfermera. Una vez obtenidos los valores de los tres observadores, se procedió a la tabulación de las cifras y a la aplicación del análisis estadístico.

\section{Resultados}

El producto de este trabajo es significativo desde el punto de vista clínico y estadístico. Al comparar las cifras de TA tomadas por el residente y el interno, se encontró una diferencia de más de $10 \mathrm{~mm} \mathrm{Hg}$ en el $15 \%$ (intervalo de confianza 95\% 0,12 0,18 ) de las cifras sistólicas y $15 \%$ (intervalo de confianza 95\% 0,12-0,18) de las diastólicas. Cuando se hizo lo propio entre el residente y la enfermera, este porcentaje ascendió a 29\% (intervalo de confianza 95\% $0,26-0,33$ ) y $30 \%$ (intervalo de confianza $95 \% 0,26-0,34)$, respectivamente.

$\mathrm{Al}$ aplicar el coeficiente de correlación de Pearson, fue evidente una mayor concordancia entre las tensiones tomadas por el interno y el residente, que entre las tomadas por el residente y la enfermera. Dicho coeficiente es una aproximación para determinar que tanta diferencia se encuentra en la precisión, observándose que el menor coeficiente de correlación de Pearson obtenido fue para la medición de la presión diastólica, que correlaciona las mediciones hechas por el residente vs. enfermera $(r=0,78)$ y el mayor coeficiente que se obtuvo fue para la determinación en la presión sistólica medida por el residente vs. interno $(r=0,91)$ (Tabla 1).

El cálculo del coeficiente de concordancia de Lin, y la representación gráfica de Band-Altman tienen en cuenta no sólo en que medida los valores obtenidos por un observador varían en función de los de otro
Tabla 1. Coeficiente de correlación de Pearsen entre los diferentes observadores en la toma de tensión arterial sistólica y diastólica medidas en $\mathbf{5 9 2}$ pacientes

Observadores y

Sistólica residente/enfermera

iastólica interno/enfermera

Sistólica interno/enfermera

iastólica residente/interno

Tabla 2. Coeficientes de concordancia de Lin entre los diferentes observadores para las presiones arteriales sistólica y diastólica en 592 pacientes

Observadores y
tipo de medición

Diastólica residente/enfermera

Sistólica residente/enfermera

Diastólica interno/enfermera

Diastólica residente/interno

Sistólica residente/interno

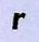

$\begin{array}{lll}0,78 & 0,74 & -0,80 \\ 0,84 & 0,84 & -0,86 \\ 0,78 & 0,75 & -0,81 \\ 0,83 & 0,80 & -0,85 \\ 0,85 & 0,83 & -0,88 \\ 0,91 & 0,90 & -0,92\end{array}$

Intervalo de confianza $95 \%$

$\%$




\section{Tabla 3. Diferencia entre los promedios de las mediciones \\ de las presiones sistólica y diastólica realizadas \\ por dos observadores en 592 pacientes}

\section{Observadores y tipo de medición}

Promedio de la diferencia

\section{Diastólica residente/enfermera}

Sistólica residente/enfermera

Diastólica interno/enfermera

Sistólica interno/enfermera

Diastólica residente/interno

Sistólica residente/interno

$\begin{array}{ll}0,573 & 15,5-16,6 \\ 0,213 & 18,4-18,8 \\ 0,660 & 15,2-16,4 \\ 0,600 & 17,6-18,8 \\ 0,080 & 12,8-13,4 \\ 0,390 & 13,6-14,4\end{array}$

Al observar que hay una diferencia de más de $10 \mathrm{~mm} \mathrm{Hg}$ en el $30 \%$ de las tomas comparando enfermera - residente y del $15 \%$ entre residente - interno, podemos deducir que la toma efectuada por el interno y la enfermera no cumplió con las especificaciones requeridas para este procedimiento. Cabe aclarar que se descartan problemas técnicos en los equipos, ya que fueron revisados y calibrados antes de iniciar el estudio.
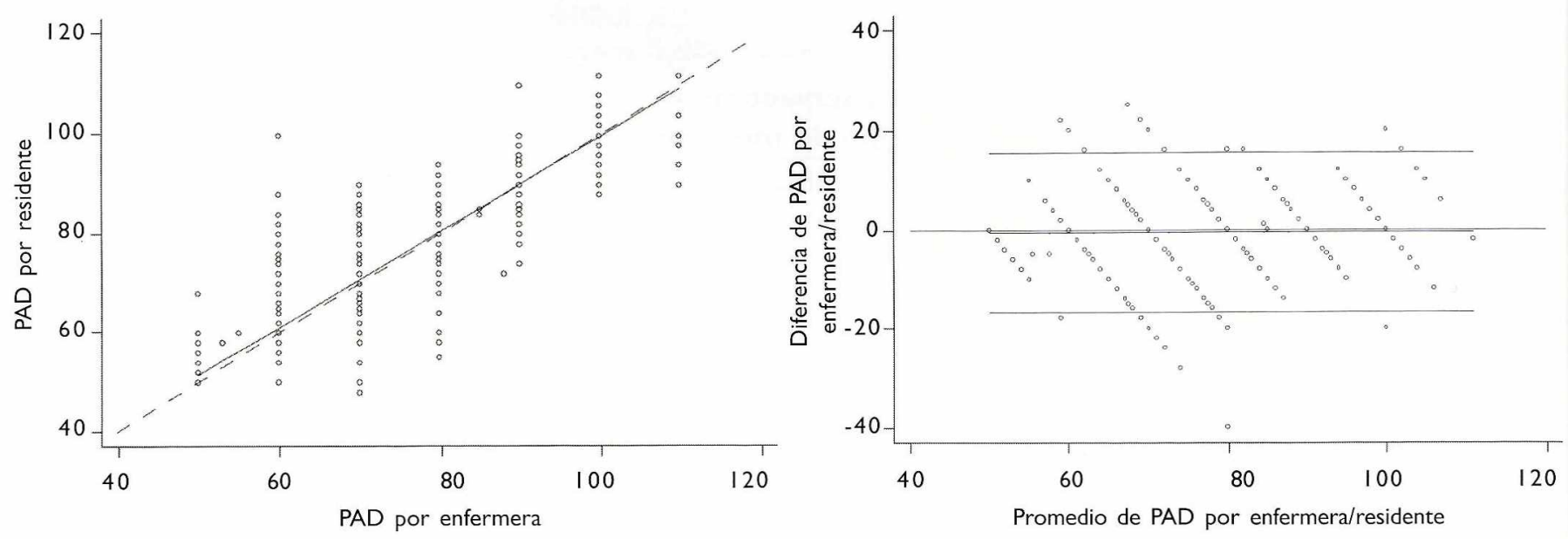

Figura I. Coeficientes de correlación y de concordancia entre las mediciones de la presión arterial diastólica residente vs. enfermera. PAD: Presión arterial diastólica.
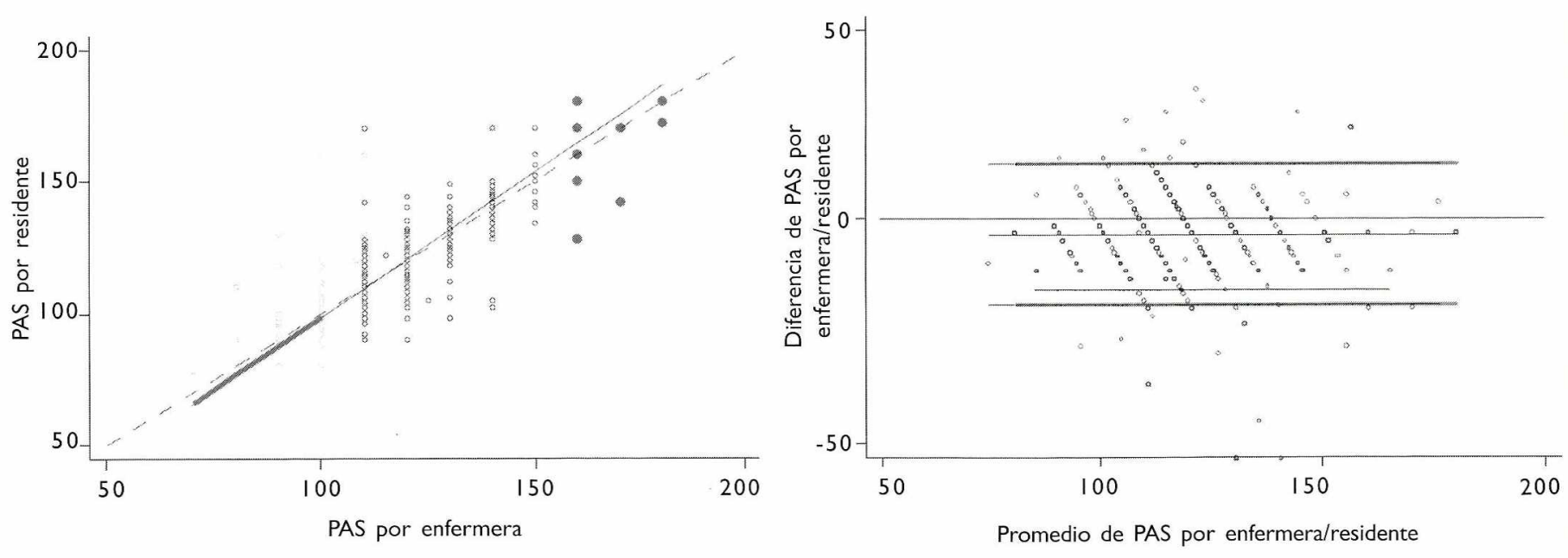

Figura 2. Coeficientes de correlación y de concordancia entre las mediciones de la presión arterial sistólica residente vs. enfermera. PAS: Presión arterial sistólica. 

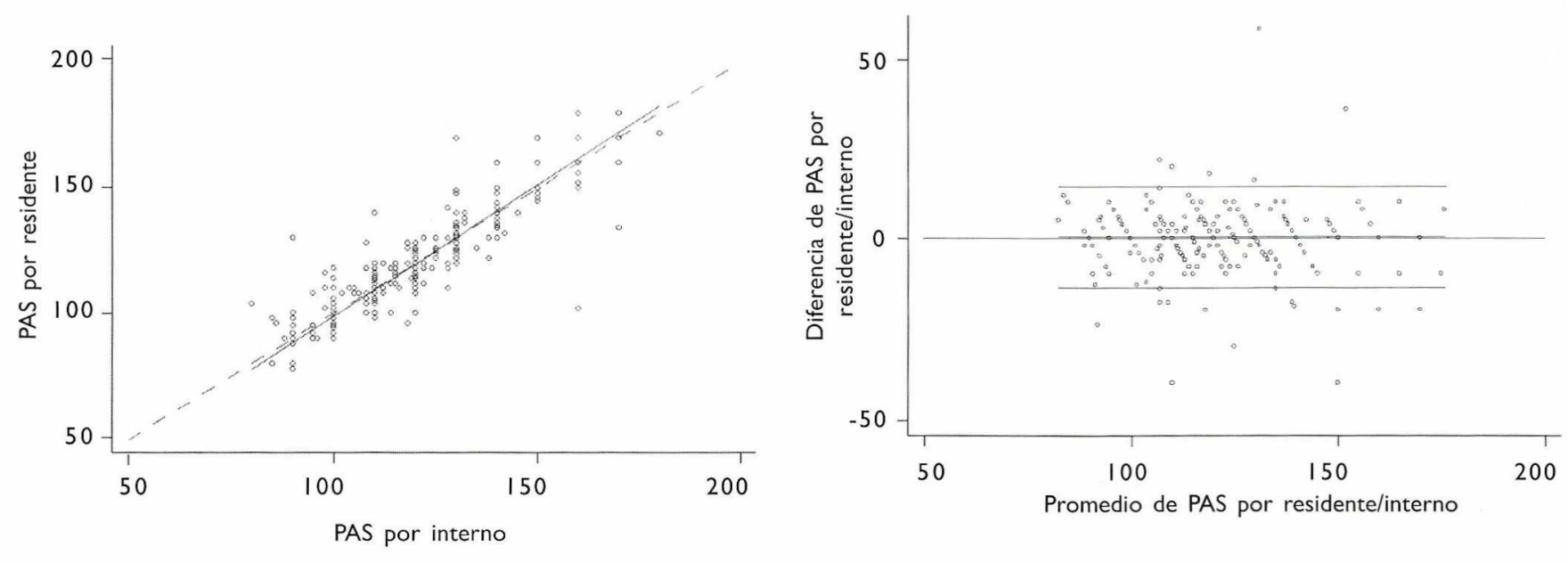

Figura 6. Coeficientes de correlación y de concordancia entre las mediciones de tensión arterial sistólica residente vs. interno. PAS: Presión arterial sistólica.

\section{Conclusiones}

Teniendo en cuenta que el residente utilizó la técnica en una forma precisa y dadas las diferencias tan marcadas en las cifras obtenidas, se deduce que la forma en que se está tomando le tensión arterial no es la adecuada. Por tanto, es imperativo instruir al personal médico y paramédico en la manera correcta de tomar la TA, pues siendo un procedimiento sencillo, la realización de éste de manera rutinaria ha hecho que se olvide la técnica correcta para una adecuada toma de la misma, conllevando a una decisión terapéutica equivocada. Esto a la postre genera un alto costo económico y a posible iatrogenia, con probables consecuencias funestas para los pacientes y médico-legales para el personal de salud.

\section{Referencilas}

1. R. Cediel. Semiología médica. Cuarta Edición. Editorial Médica Celsus.

2. G. Mejía, Semiología médica. Quinta Edición, Editorial Andina.

3. J. Latour. Las mediciones clínicas en cardiología: validez y errores de medición. Puesta al Día. Unidad de Bioestadística Clínica Hospital Ramón y Cajal.

4. H. Aguado, La cuantificación de la variabilidad en las observaciones clínicas, Med Clin Barc 1990; 95: 424 - 429.

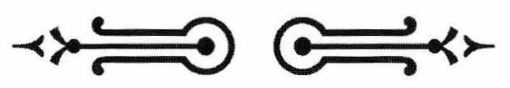

